\title{
Everolimus Associated with Activated Vitamin D for Post-Transplant Increase Epstein-Barr-Virus Load Treatment. Two Case Reports
}

\author{
Luciano Moscarelli*, Giulia Antognoli \\ Renal Unit, Careggi University Hospital, Florence, Italy. \\ Email: *moscarellil@libero.it
}

Received September $12^{\text {th }}, 2012$; revised October $20^{\text {th }}, 2012$; accepted November $4^{\text {th }}, 2012$

\begin{abstract}
Post-transplant lymphoproliferative disease (PTLD) is an uncommon but life-threatening complication of solid-organ and blood stem-cell transplants. It is caused by an uncontrolled expansion of B lymphocytes infected with Epstein-Barr virus (EBV). It responds poorly to therapy, including reduction of immunosuppression, interferon, antivirals or chemotherapy. Therefore the optimal strategy for management is currently focused on prevention. Some centers have already introduced chemoprophylaxis and/or preemptive strategies using EBV viral load as a surveillance. Some experimental studies suggest that mTOR inhibitors inhibits growth of human EBV-transformed B lymphocytes and vitamin D had an immune response to EBV. We report two cases of an increased of blood BKV viral load after renal transplantation that were successfully treated with everolimus in association with calcitriol. This report suggests that everolimus associated with calcitriol could be an effective and safe treatment for the prevention of PTLD in transplant recipients.
\end{abstract}

Keywords: Calcitriol; Everolimus; Post-Transplant Lymphoproliferative Disease; Renal Transplant

\section{Introduction}

Post-transplant lymphoproliferative disorder (PTLD), which usually represent expansion of B lymphocytes infected with Epstein-Barr virus (EBV), are a lifethreatening complication of the immunosuppressive therapy necessary to prevent graft rejection [1]. EBV is an ubiquitous human herpesvirus that has the unique ability to infect, activate, and latently persist in B lymphocytes for the lifetime of the infected patient. During primary infection, EBV transmitted via saliva infects naïve $\mathrm{B}$ cells in the tonsil. EBV drives the infected $\mathrm{B}$ cells out of the resting state to become an activated B blast and then exploits the normal pathways of $\mathrm{B}$ cell differentiation so that the $\mathrm{B}$ blast differentiates in a germinal centre to become a latently infected resting memory B cell which exist from the germinal centre and circulates in the blood [2]. Latently infected memory B cells returning to the tonsil can terminally differentiate into plasma cells, which initiates the replicative cycle with the production of infectious virus [3]. The resulting free virions infect tonsil epithelial cells where the virus replicates at high rate and is continuously shed into saliva for transmission to new hosts [4]. Newly formed virus

*Corresponding author. can also infect additional naïve B cells in the same host. EBV infection is normally kept under tight control by EBV-specific immune responses, especially by cytotoxic CD8+ T cells which eliminate proliferating and litycally infected B cells [5]. In the developing world most children become infected within the first three years of life, and EBV seropositivity reaches $100 \%$ within the first decade [6]. These early primary infections are almost always asymptomatic. In contrast, in the developed world, up to half the children are still EBV seronegative at the end of their first decade and subsequently become infected through intimate oral contact in adolescence or young adulthood [6]. As many as $50 \%$ of these delayed primary infections are symptomatic and manifest as acute infectious mononucleosis. Pharmacologic blunting of the immune system as immunosuppressive agents permits EBV-infected lymphoid cells to proliferate unabated with the risk that clonal outgrowth and frank malignant transformation may occur. Two experimental studies have suggested that rapamycin, a potent immunosuppressive drug, inhibits B-cell lymphoma in vitro [7,8]. Rapamycin is a macrolide antifungal antibiotic isolated from Streptomyces hygroscopicus, and it is the first mTOR inhibitors (mTORi) followed by sirolimus and everolimus (EVR). Rapamycin blocks the cellular cycle progression 
from phase G1 to S, by inhibition of some signal transduction pathways regulated by kinase mTOR [9]. Besides its inhibitory effects on normal cells of the immune system, rapamycin also inhibits proliferation of transformed cell lines. Various cell lines can be affected, including those of the central nervous system, liver, melanocytes, osteoblast, miogenic, renal, and connectivetissue cells, as well as T and B cells transformed. Given the data from preclinical and anecdotal reports regarding mTORi for EBV-PTLD $[7,8]$ and regarding vitamin D for immune response to EBV [10], we tested mTORi, EVR associated with calcitriol for the treatment of an increase of EBV viral load in two renal transplant recipients.

\section{Case Reports}

\subsection{Case 1}

A 46-year-old woman with unknown native kidney disease, received a renal transplant from a deceased donor in January 2010. Her immunosuppressive protocol included basiliximab (20 mg intravenously on 0 and 4th day), cyclosporine (Cya) (trough blood levels: 50 - 100 $\mathrm{ng} / \mathrm{mL}$ ), Mycophenolate mofetil (MMF) $2 \mathrm{~g} /$ day and methylprednisolone $8 \mathrm{mg} / \mathrm{day}$. The patient was discharged 15 days after transplantation (Tx) without any complications and with serum creatinine $(\mathrm{sCr})$ of 1.0 $\mathrm{mg} / \mathrm{dL}$. In June 2010, she was admitted in our institution with fever, abdominal pain, leukopenia, and sCr of 2.0 $\mathrm{mg} / \mathrm{dL}$. She was pale, without palpable lymph nodes, and with a normal chest film. Her cytomegalovirus (CMV) polymerase chain reaction (PCR) revealed $1.3 \times 10^{4}$ DNA copies $/ \mathrm{mL}$, and renal biopsy was normal. The CMV infection was treated with ganciclovir. Immunosuppressive therapy was reduced (MMF was discontinued). CMV PCR became negative within 14 days of administration of ganciclovir. After one month CMV PCR was negative and EBV PCR was 800 copies $/ \mathrm{mL}$. At Tx EBV serological status was donor positive and recipient negative. After two weeks EBV PCR had a great increase $\left(4 \times 10^{5}\right.$ copies $\left./ \mathrm{mL}\right)$. Cerebral computed tomography
(CT) scan, thoracic CT scan, abdominal CT scan, and bone marrow biopsy were negative. MMF was discontinued definitively, and EVR was started at $3.75 \mathrm{mg} /$ day (aiming to avoid the relapses of EBV PCR increase and to maintain graft function) associated with calcitriol 1 $\mathrm{mg} /$ day for oral administration. No adverse effects were observed. At present, 24 months after treatment, she is asymptomatic without any relevant increase of the EBV viral load (Figure 1), with sCr of $1.1 \mathrm{mg} / \mathrm{dL}$. Her immunosuppressive regimen comprises CyA (trough blood levels 50 - $70 \mathrm{mg} / \mathrm{ml}$ ) EVR (blood levels 8 - $10 \mathrm{ng} / \mathrm{ml}$ ) and methylprednisolone (4 mg/day).

\subsection{Case 2}

A 52-year-old men with end-stage renal disease secondary to interstitial nephritis, received a renal transplant from deceased donor in May 2009. He presented with delayed graft function requiring haemodialysis for two weeks. His renal biopsy on day 7 showed acute rejection grade I and acute tubular necrosis (Banff classification). He received $500 \mathrm{mg} /$ day of methylprednisolone for 3 alternate days. He was discharged on day 28 as asymptomatic, and with sCr of $2.0 \mathrm{mg} / \mathrm{dL}$. His immunosuppressive protocol included basiliximab (20 mg intravenously on 0 and 4th day), Cya (trough blood levels: 50$100 \mathrm{ng} / \mathrm{mL}$ ), MMF $2 \mathrm{~g} /$ day and methylprednisolone 8 $\mathrm{mg} /$ day. At one year after Tx, he was admitted in our institution because of a skin lesion left arm unspecified and an increased of sCr. The biopsy of skin lesion, showed a diffuse polymorphic CD20+ B-cell lymphoma heavily infiltrated with T cells. Thoracic CT scan, abdominal CT scan, and bone marrow biopsies were negative. Clinical staging was IIe (Ann Arbor). Age-adjusted IPI was 2 (high-intermediate risk category). EBV PCR was $2 \times 10^{5}$ copies/mL. MMF was discontinued and the patient was treated with 4 weekly doses of $375 \mathrm{mg} / \mathrm{m}^{2}$ of rituximab. One month after, only a minimal residual skin lesion was detected. EBV PCR was $<500$ copies $/ \mathrm{mL}$. After 4 weeks EBV PCR increased considerably $(1.5 \times$ $10^{5}$ copies $\left./ \mathrm{mL}\right)$. MMF was discontinued definitively, and

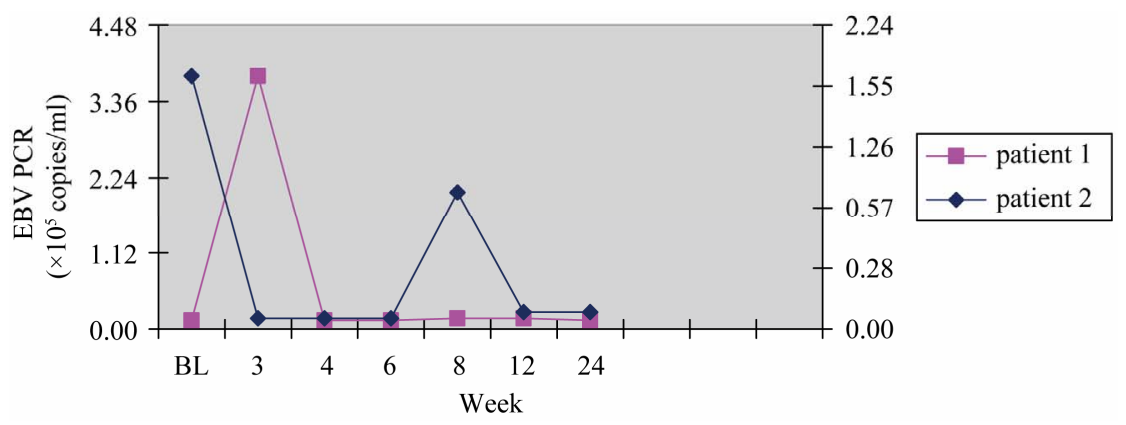

Figure 1. EBV PCR by week of treatment. 
EVR was started at $4.0 \mathrm{mg} /$ day (aiming to avoid the relapses of EBV PCR increase and to maintain graft function) associated with calcitriol $1 \mathrm{mg} /$ day for oral administration. No adverse effects were observed. At present, 22 months after treatment, he is asymptomatic without any relevant increase of the EBV viral load (Figure 1). His immunosuppressive regimen comprises CyA (trough blood levels $50-70 \mathrm{mg} / \mathrm{ml}$ ) EVR (blood levels 8 - 10 $\mathrm{ng} / \mathrm{ml}$ ) and methylprednisolone (4 mg/day). At the Tx EBV serological status was donor positive and recipient positive.

In both patients baseline EBV PCR were obtained via Light-Cycler PCR and followed weekly.

Both patients have approved the publication of this case report.

\section{Discussion}

In this paper, we describe two renal transplant recipients with an increase of EBV plasma viral load successfully treated with EVR associated with calcitriol. PTLDs are a recognized complication of the immunosuppression required to prevent allograft rejection, occurring in $1 \%$ $20 \%$ of recipients of solid organ transplants. PTLDs comprise a whole spectrum of lymphoproliferative disorders ranging from a polyclonal atypical lymphoid hyperplasia to a monoclonal, overtly malignant B cell lymphoma [11]. The EBV genome is found in the majority of $B$ cells. PTLD occurring early (within the first year) after solid organ Tx and EBV infection is believed to play a major pathophysiologic role in their development. A defect in T-cell regulation allows uncontrolled proliferation of B (90\% of cases) or other T lymphocytes in response to a viral infection, particularly EBV (90\% - 95\% of PTLDs are positive for EBV) as a consequence of prolonged administration of immunosuppressive drugs [12]. Although relatively uncommon, the risk of developing lymphoma after Tx has been reported to be 28 - 49 times higher than in the general population, and it is believed to be responsible for $16 \%$ of tumours in transplant patients $[13,14]$. The prevalence ranges from $1 \%-10 \%$. It depends on many factors, including allograft type, Epstein-Barr virus serological status before Tx, adult versus pediatric population, underlying disease, and degree and duration of immunosuppression. Additional risk factors include lymphoid cell phenotype at Tx, CMV infection, and cytokine promoter gene polymorphism [12-14]. For same time PTLD is asymptomatic until no symptoms depending on the organ affected. PTLD can involve any abdominal visceral organ although the transplanted kidney is the most common primary site (47\% - 70\%) [15] with presenting features including graft dysfunction, fever, and hydronephrosis secondary to ureteric obstruction.
The next most common extranodal site is the gastrointestinal tract. Other sites PTLD has been reported to affect include the sino-nasal cavity, central nervous system, skin and lymph nodes in isolation. Few treatment options exist when the disease is progressive despite reduction of immunosuppression, antiviral agents, monoclonal B-cell antibodies, cytotoxic chemotherapy, or adoptive transfer of EBV-specific cytotoxic T lymphocytes [14]. Less advanced forms of PTLD respond to a decrease in the dose of the immunosuppressive agents. However, lowering the drug dose jeopardizes survival of the graft. In the absence of reliably effective therapy for all stages of PTLD, the optimal strategy for management is currently focused on prevention. Some centers have already introduced chemoprophylaxis and/or preemptive strategies using EBV viral load as a surveillance. EVR has a high inhibittory effect on in vitro growth of six different PTLD-like EBV + lymphoblastoid B-cells lines. Similarly to normal T-cells, EVR blocked cell-cycle progression in PTLDlike B-cells in the early phase. This drug also had a profound inhibitory effect on the growth of PTLD-like EBV + B-cells xenotransplanted into severe combined immunodeficiency mice [7]. The vitamin D system had multiple physiological and pharmacological effects mediated by action of the vitamin D receptors (VDRs). Recently, VDR activators (VDRA) have been shown to obstruct the cell replication and have an immunomodulatory properties. An important observation was reported which suggested that toll-like receptors (TLR) activation of human macrophages upregulated expression of VDRs and vitamin D-1-hydroxylase genes, leading to induction of the antimicrobial peptide [16] this suggests an association of TLR and vitamin D-mediated innate immunity [16] which represent a first line of defense against infection. To our knowledge, this is the first report of this clinical approach to an EBV viral load increase. Because only two patients with limited follow-up are described, the role of this combination on efficacy of increase of EBV PCR treatment need to be established, and this indicate that a larger prospective trial to test this drugs association is warranted. It would also be useful to establish whether this drug association can be effective for the prevention of PTLD in transplant recipients.

\section{Conclusion}

PTLD is an uncommon but life-threatening complication of solid-organ and blood stem-cell transplants. It responds poorly to therapy, including reduction of immunosuppression, interferon, antivirals or chemotherapy. Therefore the optimal strategy for its management is currently focused on prevention and preemptive strategies using EBV viral load as a surveillance may be useful. 
In this report we have seen the usefulness of drug combination from EVR and calcitriol on increase of EBV PCR but only two patients not allow us to determine its real effectiveness.

\section{Acknowledgements}

The Author thanks Anna Morra for her technical assistance.

\section{REFERENCES}

[1] S. M. Cockfield, "Identifying the Patient at Risk for Post-Transplant Lymphoproliferative Disorder," Transplant infectious Disease, Vol. 3, No. 2, 2001, pp. 70-78. doi:10.1034/j.1399-3062.2001.003002070.x

[2] D. A. Thorley-Lawson and A. Gross, "Persistence of the Epstein-Barr Virus and the Origins of Associated Lymphomas," The New England Journal of Medicine, Vol. 350, No. 13, 2004, pp. 1328-1337. doi:10.1056/NEJMra032015

[3] L. L. Laichalk and D. A. Thorley-Lawson, "Terminal Differentiation into Plasma Cells initiates the Replicative Cycle of Epstein-Barr Virus in Vivo,” Journal of Virology, Vol. 79, No. 2, 2005, pp. 1296-1307. doi:10.1128/JVI.79.2.1296-1307.2005

[4] V. Hadinoto, M. Shapiro, C. C. Sun, et al., "The Dynamics of EBV Shedding Implicate A Central Role for Epithelial Cells in Amplifying Viral Output," PLoS Pathogens, Vol. 5, No. 7, 2009, p. 1.

[5] A. D. Hislop, G. S. Taylor, D. Sauce, et al., "Cellular Responses to Viral infection in Humans: Lessons from Epstain-Barr Virus,” Annual Reviews of Immunology, Vol. 25, No. 1, 2007, pp. 587-617. doi:10.1146/annurev.immunol.25.022106.141553

[6] A. B. Rickinson and E. Kieff, "Epstein-Barr Virus,” In: D. M. Knipe and P. M. Howley, Eds., Fields Virology, Lippincott Williams \& Wilkins, Philadelphia, 2001, pp. 2575-2627.

[7] M. Majewski, M. Korecka, P. kossev, et al., "The Immunosuppressive Macrolide RAD Inhibits Growth of Human Epstein-Barr Virus-Transformed B Lymphocytes in Vitro and in Vivo: A Potential Approach to Prevention and Treatment of Posttransplant Lymphoproliferative Disor- ders,” Proceedings of the National Academy of Sciences, Vol. 97, No. 8, 2000, pp. 4285-4290

[8] S. Muthukkumar, T. M. Ramesh and S. Bondada, "Rapamycin, a Potent Immunosuppressive Drug, Causes Programmed Cell Death in B Lymphoma Cells,” Transplantation, Vol. 60, No. 3, 1995, pp. 264-270. doi:10.1097/00007890-199508000-00010

[9] S. N. Seghal, J. S. Camardo, J. A. Scarola, et al., "Rapamycin (Sirolimus, Rapamune)," Current Opinion in Nephrology and Hypertension, Vol. 4, No. 6, 1995, pp. 482-487. doi:10.1097/00041552-199511000-00004

[10] T. Holmoy, "Vitamin D Status Modulates the Immune Response to Epstein Barr Virus: Synergistic Effect of Risk Factors in Multiple Sclerosis,” Medical Hypotheses, Vol. 70, No. 1, 2008, pp. 66-69. doi:10.1016/j.mehy.2007.04.030

[11] Y. Dror, M. Greenberg, G. Taylor, et al., "Lymphoproliferative Disorders after Organ Transplantation in Children,” Transplantation, Vol. 67, No. 7, 1999, pp. 990-998. doi:10.1097/00007890-199904150-00010

[12] R. Shapiro, M. Nalesnik, J. Mc Cauley, et al., "Posttransplant Lymphoproliferative Disorders in Adult and Pediatric Renal Transplant Patients Receiving Tacrolimus-Based Immunosuppression,” Transplantation, Vol. 68, No. 12, 1999, pp. 1851-1854. doi:10.1097/00007890-199912270-00006

[13] B. S. Younes, S. V. Mc Diarmid, M. G. Martin, et al., "The Effect of Immunosuppression on Posttransplant Lymphoproliferative Disease in Pediatric Liver Transplant Patients,” Transplantation, Vol. 70, No. 1, 2000, pp. 94-99.

[14] J. K. Preiksaitis and S. Keay, "Diagnosis and Management of Post-Transplant Lymphoproliferative Disorder in Solid-Organ Transplant Recipients," Clinical Infectious Diseases, Vol. 33, No. S1, 2001, pp. S38-S46.

[15] C. E. Kew, R. Lopez-Ben and J. K. Smith, "Posttransplant Lymphoproliferative Disorder Localized near the Allograft in Renal Transplantation,” Transplantation, Vol. 69, No. 5, 2000, pp. 809-814.

[16] P. T. Liu, S. Stenger, H. Y. Li, et al., "Toll-Like Receptor Triggering of a Vitamin D-Mediated Human Antimicrobial Response,” Science, Vol. 311, No. 5768, 2006, pp. 1770-1773. 